Trinken ist nicht gleich Trinken

\title{
Nierensteine mögen es zuckersüß
}

— Die Flüssigkeitsaufnahme insgesamt zu erhöhen, senkt das Risiko für Nierensteine. Bei manchen Getränken verhält es sich jedoch genau umgekehrt. Zuckerhaltige Limonaden taugen einer Analyse von drei prospektiven Kohortenstudien mit 194.000 Studienteilnehmern zufolge nicht zur Nephrolithiasis-Prävention, der Konsum ist vielmehr mit einem erhöhten Steinrisiko assoziiert.

Probanden, die täglich mindestens eine zuckerhaltige Cola tranken, hatten ein um 23\% höheres Steinrisiko als Personen, die seltener als einmal wöchentlich ein solches Getränk zu sich nahmen. Bei anderen Limonaden mit Zucker war das Risiko bei täglichem Konsum sogar um 33\% erhöht. Kein signifikanter Zusammenhang mit dem
Steinrisiko zeigte sich dagegen bei Limonaden, die statt Zucker Süßstoff enthielten. Das spricht dafür, dass die in zuckersüßen Brausegetränken enthaltene Fruktose die Steinbildung begünstigt. Fruktose steigert nachweislich die Ausscheidung von Kalzium, Oxalat und Harnsäure über den Urin. In der Analyse wurde außerdem für verschiedene Getränke wie Kaffee, Tee, Bier, Wein und Orangensaft bestätigt, dass mit steigendem Konsum das Risiko für Nierensteine sinkt. Dass Orangensaft der Steinbildung entgegenwirkt, mag angesichts des hohen Fruktosegehaltes überraschen; wahrscheinlich wird dieser aber durch die günstigen Effekte des reichlich vorhandenen Kaliumzitrats mehr als wettgemacht.

Dr. Beate Schumacher

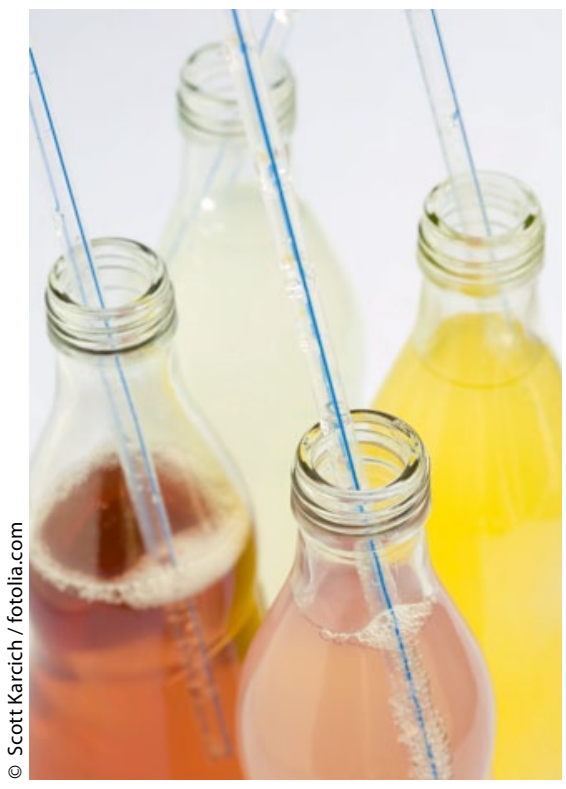

Zuckerhaltige Limonaden: für die Steinprävention ungeeignet

\section{Eingeschränkte Fertilität}

\section{Jeder dritte Diabetiker mit retrograder Ejakulation}

— Neuropathische Störungen sind eine mögliche Ursache der retrograden Ejakulation (RE). Und so liegt es eigentlich nahe, dass Diabetiker von RE betroffen sein können. Obgleich bei bekannter RE der Verdacht auf das Vorliegen eines Diabetes besteht, ist über die allgemeine Prävalenz der RE bei Diabetikern wenig bekannt. Ein dänisches Team von Fertilitäts- und Sexualmedizinern hat nun im Zuge einer Studie versucht, die Wissenslücke zu schließen. Zwar nahmen nur wenige Probanden an der Studie teil: 26 Diabetiker und 16 Kontrollpersonen, im Mittel zwischen 40 und 50 Jahre alt. Um statistisch stichfest zu belegen, dass Diabetiker erstaunlich häufig eine RE haben, reichte die Probandenzahl allerdings aus: Bei neun der 26 Studienteilnehmer mit Diabetes $(34,6 \%)$ wurde die Samenflüssigkeit zumindest teilweise in Richtung Harnblase ausgestoßen. Die Suche nach Faktoren, die mit Diabetes und RE assoziiert sind, verlief ergebnislos.

Die Autoren empfehlen bei Diabetikern, die eine Aspermie oder sehr kleine Ejakulatvolumina haben, eine postejakulatorische Urinanalyse. Falls die Spermatozoenzahl ausreiche, könnten aus solchem Urin eventuell sogar Spermien für die künstliche Befruchtung gewonnen werden. Ein hierfür förderliches Urinmilieu lässt sich bei Bedarf durch geeignete Trinklösungen herstellen.

Dr. Robert Bublak
Copenhagen City Heart Study

\section{Läufer leben länger - wenn sie nicht übertreiben}

— Leben Langstreckenläufer gefährlich oder gesünder? Um das allgemeine Mortalitätsrisiko von Joggern zu erfassen, wurden knapp 18.000 gesunde Männer und Frauen zwischen 20 und 98 Jahren in der Zeit von 1976 bis 2003 im Rahmen der Copenhagen City Heart Study untersucht und zu ihren körperlichen Aktivitäten befragt [Schnohr et al. Am J Epidemiol 2013; 177: 683-9]. Für Läufer errechneten die Autoren altersadaptiert ein um 44\% reduziertes Gesamtmortalitätsrisiko. Der Gewinn an zusätzlicher Lebenszeit schwankte, je nach Kofaktoren zwischen 3,1 und 5,6 Jahren bei Frauen und zwischen 2,6 und 6,2 Jahren bei Männern. Den Subanalysen zum Einfluss von Häufigkeit, Dauer und Intensität der Trainingseinheiten mangelte es zwar etwas an statistischer Aussagekraft, sie sind aber dennoch erwähnenswert: So senkte schon eine Laufzeit von unter einer Stunde pro Woche das Sterberisiko gegenüber den Nichtläufern um $32 \%$ und von 1-2,4 Stunden um $42 \%$. Doch dann drehte sich die Kurve: Bei Trainingszeiten zwischen 2,5 und 4 Stunden wurden nur noch $21 \%$ gewonnen und bei mehr als vier Stunden noch $14 \%$.

Bei der Trainingshäufigkeit ergab sich ein ähnliches Bild: Bis zu dreimal Joggen pro Woche brachte $60 \%$ Risikoreduktion. Wer häufiger die Laufschuhe schnürte, hatte dagegen ein erhöhtes Sterberisiko von $24 \%$ gegenüber den Lauffaulen. In die gleiche Kerbe schlug auch das Ergebnis der dritten Subanalyse: Langsam laufen schien den meisten Erfolg zu bringen, denn Jogger dieser Gruppe verringerten ihr Sterberisiko um 63\%. Wer dagegen schnell lief, schnitt letztlich sogar schlechter ab als die Nichtläufer.

Dr. Christine Starostzik 